06,11

\title{
Акустические аномалии в твердых растворах $\mathrm{SrTiO}_{3}-\mathrm{BiFeO}_{3}$
}

\author{
(C) E. Смирнова ${ }^{1}$, A. Сотников ${ }^{1,2}$, H. Зайцева ${ }^{1}$, H. Schmidt ${ }^{2}$ \\ ${ }^{1}$ Физико-технический институт им. А.Ф. Иофффе, \\ Санкт-Петербург, Россия \\ ${ }^{2}$ Leibniz Institute for Solid State and Materials Research Dresden, \\ Dresden, Germany \\ E-mail: esmirnoffa@gmail.com
}

(Поступила в Редакцию 5 июня 2017 г.)

Представлены результаты акустического исследования твердых растворов $\mathrm{SrTiO}_{3}-\mathrm{BiFeO}_{3}$ в диапазоне температур от 100 до $650 \mathrm{~K}$. Измерение скорости и затухания продольной ультразвуковой моды на частоте $10 \mathrm{MHz}$ проводилось эхо-импульсным методом. Наблюдаемые аномалии скорости и затухания коррелируют с максимумами диэлектрической проницаемости в температурном диапазоне релаксорного состояния. Кроме того, выявлены пики затухания в температурном диапазоне 400-600 K, определяющие характерные для релаксоров температуры Бернса и $\mathrm{T}^{*}$. Полученные результаты позволили уточнить фазовую диаграмму системы твердых растворов $\mathrm{SrTiO}_{3}-\mathrm{BiFeO}_{3}$.

DOI: 10.21883/FTT.2018.01.45296.181

\section{1. Введение}

Твердые растворы, обладающие релаксорными свойствами, широко используются для изготовления конденсаторов, электромеханических и электрооптических устройств, а также рассматриваются в качестве перспективных электрокалорических материалов [1-6]. Релаксорное поведение наблюдается как у твердых растворов, одной из компонент которых является соединениерелаксор, так и у мультикомпонентных составов, компонентами которых являются обычные сегнетоэлектрики. Особое место среди таких твердых растворов занимает система $(1-x) \mathrm{SrTiO}_{3}-x \mathrm{BiFeO}_{3} . \mathrm{SrTiO}_{3}$ (STO) относится к виртуальным сегнетоэлектрикам, в которых сегнетоэлектрическая фаза подавляется квантовыми флуктуациями (нулевыми колебаниями) вплоть до самых низких температур [7-10]. В случае STO предполагается, что, в дополнение к квантовым флуктуациям, тетрагональные искажения решетки перовскита $A B \mathrm{O}_{3}$, связанные с антиферродисторсионным AFD переходом из кубической $P m 3 m$ фазы в неполярную тетрагональную I4/ $\mathrm{mcm}$ фазу при $110 \mathrm{~K}$, также влияют на подавление сегнетоэлектрического перехода [11]. Небольшое количество изовалентных примесей может индуцировать в STO низкотемпературный сегнетоэлектрический переход или переход в стеклоподобное полярное состояние. При $A$-замещении иона $\mathrm{Sr}$ в решетке перовскита на $\mathrm{Ba}[12-14], \mathrm{Pb}$ [15], $\mathrm{Ca}[16-19], \mathrm{Cd}[20]$ и $\mathrm{Bi}$ [21] наблюдается обычный сегнетоэлектрический переход или релаксорное сегнетоэлектрическое состояние.

$\mathrm{B}$ семействе сегнетомагнетиков $\mathrm{BiFeO}_{3}$ (BFO) выделяется высокими температурами Кюри $T_{C} \approx 1093 \mathrm{~K}$ и Нееля $T_{\mathrm{N}} \approx 637 \mathrm{~K}$ [22-24]. Сообщалось о чрезвычайно высоких величинах спонтанной поляризации около $60-100 \mu \mathrm{Ccm}^{-2}$ при низком коэрцитивном поле $12 \mathrm{KV} / \mathrm{cm}[25,26]$ для монокристаллов, керамики $\left(40 \mu \mathrm{Ccm}^{-2}\right)$ [27] и пленок феррита висмута (вплоть до $\left.158 \mu \mathrm{Ccm}^{-2}\right)[28,29]$ при комнатной температуре. Введение редкоземельных магнитоактивных ионов в положение иона Ві в керамических твердых растворах на основе BFO приводит к возникновению слабого ферромагнетизма и появлению линейного магнитоэлектрического эффекта [30-32]. Отмечается также, что замещение $\mathrm{Fe}$ на ионы переходных металлов в поликристаллических пленках феррита висмута приводит к увеличению поляризации и намагниченности [33]. Перечисленные выше свойства указывают на перспективность использования BFO как базового компонента твердых растворов при создании новых материалов с полезными для различных применений свойствами. В системе $(1-x) \mathrm{STO}-x \mathrm{BFO}$ нами было обнаружено возникновение полярного состояния, которое характеризуется максимумами диэлектрической проницаемости с температурами $T_{m}$, eе дисперсией и петлями поляризации $[34,35]$. При самых малых концентрациях зависимость $T_{m}(x)$ описывается соотношением $T_{m}=A\left(x-x_{c}\right)^{1 / 2}$ с критической концентрацией $x_{c} \approx 0.002$, что является величиной типичной для виртуальных сегнетоэлектриков, в которых сегнетоэлектрический фазовый переход индуцируется примесями $[12,14,17]$. Измеренные при различных температурах спектры диэлектрической проницаемости и построенные на их основе зависимости $\varepsilon^{\prime}(T)$ на разных частотах для концентраций $0<x \leq 0.2$ демонстрируют поведение, типичное для релаксоров, а именно размытые максимумы диэлектрической проницаемости со сдвигом температуры максимума $T_{m}$ в область более высоких температур с увеличением частоты измерений [34,35]. Однако определения частотной зависимости диэлектрической проницаемости и $T_{m}$ недостаточно для описания релаксорного состояния. В настоящее время надежно установлено, что основные свойства релаксоров проявляются в существовании еще двух характерных темпе- 

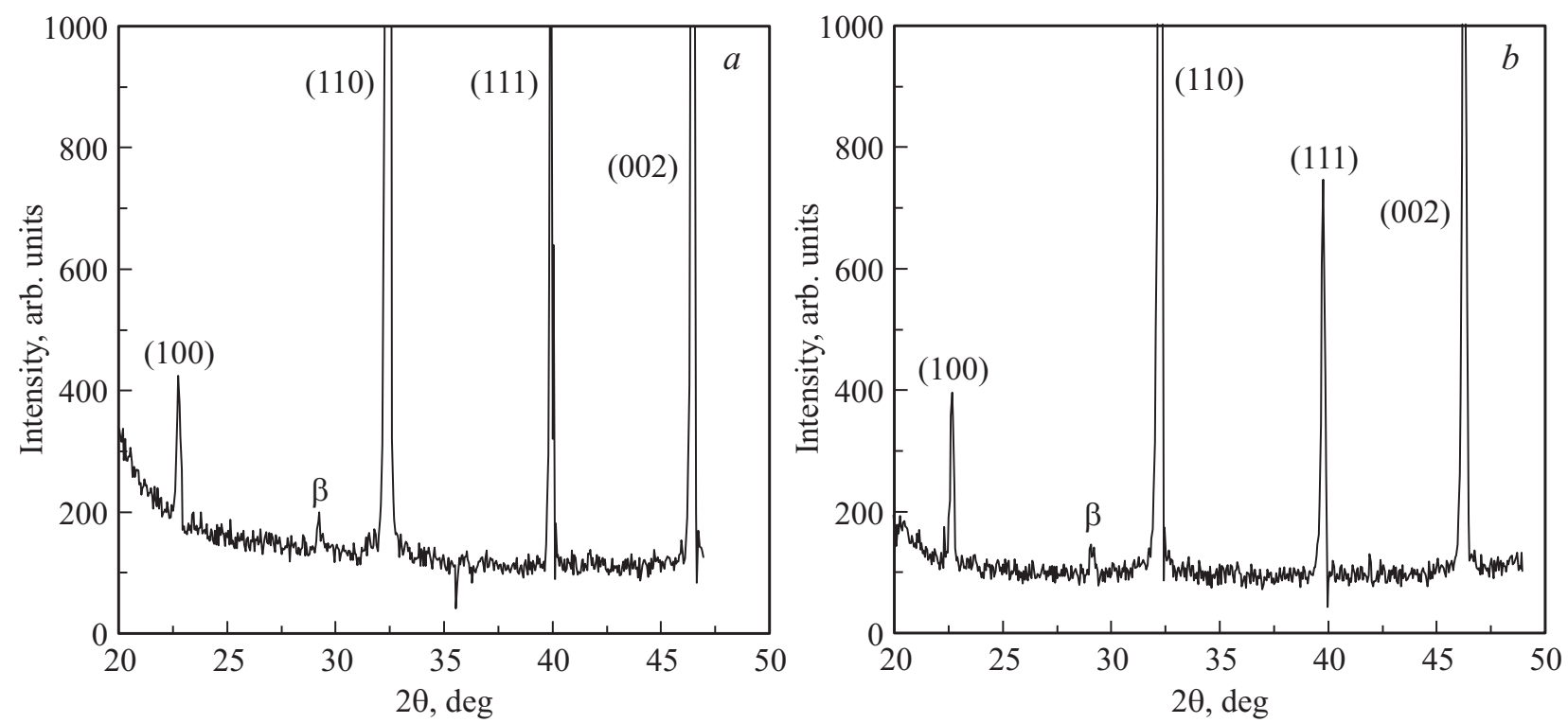

Рис. 1. Рентгеногрммы твердых растворов $\left.(1-x) \mathrm{SrTiO}_{3}-x \mathrm{BiFeO}_{3}: a\right) x=0.1$ и $\left.b\right) x=0.3$, в интервале углов от 10 до $50^{\circ}$ по $2 \theta$.

ратур. Прежде всего, это температура Бернса $T_{\mathrm{B}}$, при которой, согласно общепризнанной модели, происходит зарождение полярных областей PNR в параэлектрической матрице [36-38]. Температура отклонения обратной величины диэлектрической проницаемости от закона Кюри-Вейсса $T_{d}$ рассматривалась в качестве температуры Бернса $[39,40]$. Изменения параметра решетки и отклики акустической эмиссии были обнаружены при температуре Бернса в наиболее изученном релаксоре магнониобате свинца $(\mathrm{PMN})$ и для монокристаллов семейства цинкониобата свинца $[41,42]$. Пики затухания ульразвуковой волны при $T_{\mathrm{B}}$ наблюдались в $\mathrm{PMN}$ и твердых растворах на его основе, а также в релаксорах с магнитным упорядочением [43,44]. При интерпретации результатов упругого рассеяния нейтронов [45-47], акустической эмиссии и затухания ультразвуковых волн [41-44,48-51] было высказано предположение о существовании еще одной характерной температуры $T^{*}$, определенной в диапазоне температур 400-500 K и характеризующей сегрегацию PNR. Следует заметить, что $T_{m}<T^{*}<T_{\mathrm{B}}$.

Целью настоящего исследования было получение информации о релаксорном состоянии в системе твердых растворов $(1-x) \mathrm{STO}-x \mathrm{BFO}$ и коррекция фазовой диаграммы системы с учетом полученных результатов.

\section{2. Эксперимент}

Образцы твердых растворов $(1-x) \mathrm{STO}-x \mathrm{BFO}$ $(0.1 \leq x \leq 0.3)$ изготавливались по обычной керамической технологии. В качестве исходных реактивов использовались оксиды $\mathrm{TiO}_{2}, \mathrm{Fe}_{2} \mathrm{O}_{3}, \mathrm{Bi}_{2} \mathrm{O}_{3}$ и карбонат стронция $\mathrm{SrCO}_{3}$. Синтез и спекание керамики проводились в платиновых тиглях при температуре $1100^{\circ} \mathrm{C}$ в течение $24 \mathrm{~h}$ и температурах $1320-1300^{\circ} \mathrm{C}$ в течение одного часа соответственно. Плотность полученных образцов составляла 92-94\% от рентгеновской плотности. Для проведения рентгеноструктурных исследований синтезированных образцов использовался рентгеновский дифрактометр ДРОН-3 с излучением $\mathrm{Cu} K_{\alpha}, \lambda=$ $=1.54178 \AA$, Ni-фильтр, $38 \mathrm{kV}, 18 \mathrm{~mA}$. Сканирование проводилось в интервале углов $2 \theta$ от 10 до $160^{\circ}$.

Диэлектрические свойства измерялись с помощью анализатора импедансов Solartron SI 1260 в интервале частот от $10 \mathrm{~Hz}$ до $1 \mathrm{MHz}$ при температурах от 90 до 400-600 K. Амплитуда измерительного поля составляла $1 \mathrm{~V} / \mathrm{cm}$. Образцы имели форму дисков диаметром $8 \mathrm{~mm}$ и толщиной $0.5-1 \mathrm{~mm}$. В качестве электродов использовалась серебряная паста, которая вжигалась в образцы при температуре около $500^{\circ} \mathrm{C}$. Скорость и затухание продольной акустической волны $V_{L}$ в зависимости от температуры измерялась ультразвуковым эхо-импульсным методом с помощью установки RITEC Advanced Ultrasonic Measurement System RAM-5000 на частоте $10 \mathrm{MHz}$. Образцы для акустических измерений представляли собой керамические бруски размером $5 \times 5 \times 8 \mathrm{~mm}$. Температурные измерения в диапазоне от 100 до $350 \mathrm{~K}$ проводились в криостате фирмы Oxford Instruments, а в диапазоне от 300 до $650 \mathrm{~K}$ в печи фирмы Carbolite при стабилизации температуры с точностью $0.1 \mathrm{~K}$ или в режиме охлаждения со скоростью $1 \mathrm{~K} / \mathrm{min}$.

\section{3. Экспериментальные результаты и их обсуждение}

Рентгеноструктурный анализ при комнатной температуре показал, что синтезированные составы имеют кубическую структуру. В качестве примера, на рис. 1 

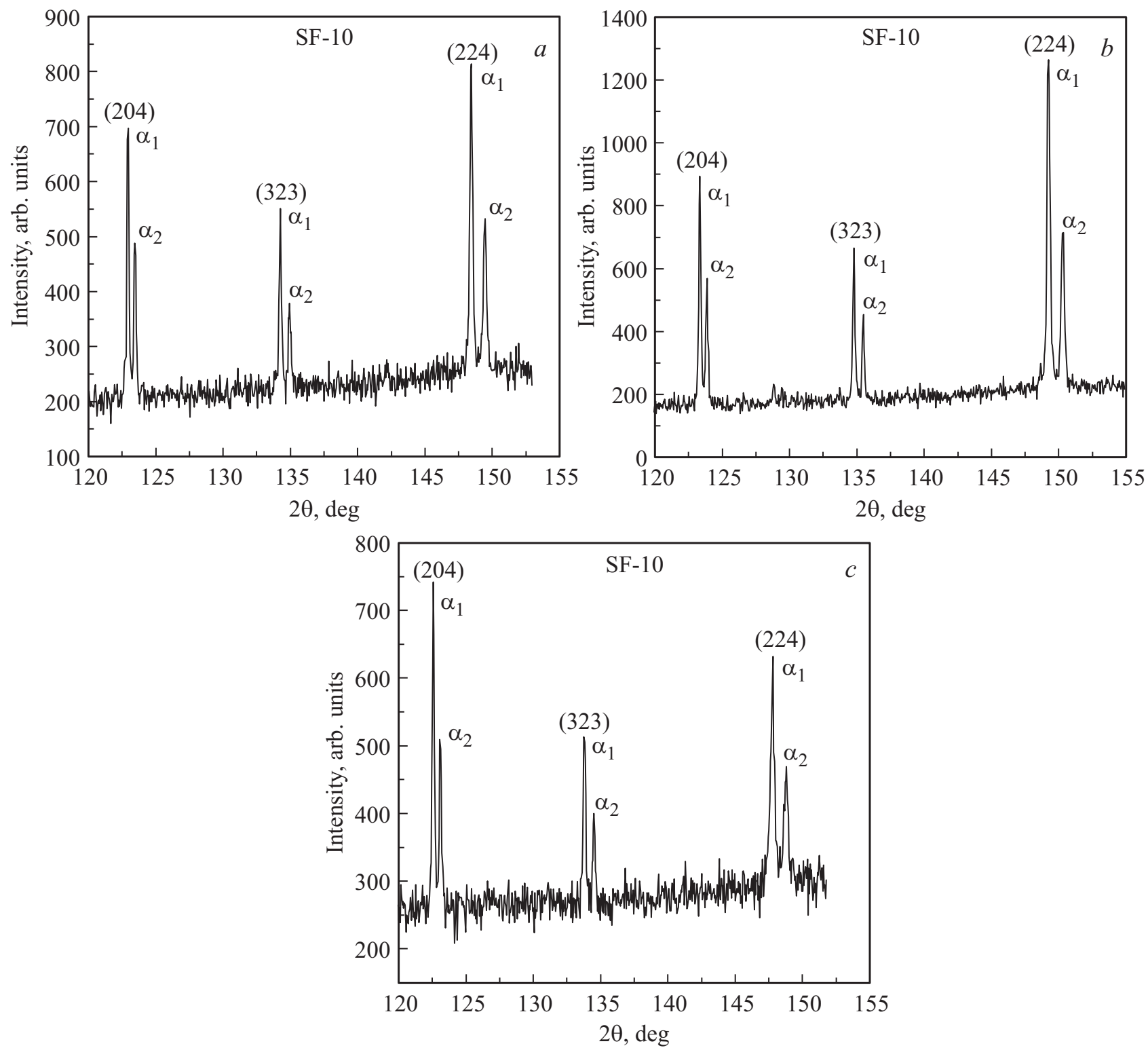

Рис. 2. Рентгеногрммы твердых растворов $\left.\left.(1-x) \mathrm{SrTiO}_{3}-x \mathrm{BiFeO}_{3}: a\right) x=0.1, b\right) x=0.2$ и $\left.c\right) x=0.3$, в интервале углов от 120 до $155^{\circ}$ по $2 \theta$.

приведены рентгенограммы в интервале углов от 10 до $50^{\circ}$ по $2 \theta$ для составов с концентрацией феррита висмута 0.1 и 0.3 . Из полученных экспериментальных данных следует, что все образцы являются однофазными со структурой перовскита. На рис. 2 приведены рентгенограммы исследованных образцов в интервале углов от 120 до $155^{\circ}$ по $2 \theta$. Этот интервал углов наиболее пригоден для определения параметров кристаллической решетки. Значения параметров варьируются от $a=3.9126 \pm 0.0003 \AA(x=0.1)$ до $a=3.9270 \pm 0.0003 \AA(x=0.3)$. Кроме того, так как дифракционные рефлексы (204), (323), (224) расщепляются только на 2 пика: $\alpha_{1}$ и $\alpha_{2}$ в соотношении $2: 1$, можно сделать вывод, что все три состава являются кубическими. В противном случае отражения расщепились бы на большее количество рефлексов, что характерно для других сингоний [52].

На рис. 3, в качестве примера, приведен фрагмент спектров действительной части диэлектрической проницаемости $\varepsilon$ для состава с концентрацией $x=0.3$. Полученные спектры диэлектрической проницаемости подтверждают типичное для релаксоров поведение, рассмотренное нами в работе [35]. Температуры размытых максимумов диэлектрической проницаемости $T_{m}$ на частоте $1 \mathrm{KHz}$ варьируются от $215 \mathrm{~K}(x=0.1)$ до $360 \mathrm{~K}(x=0.3)$. Обращает на себя внимание тот факт, что при повышении концентрации феррита висмута и соответственно изменении $T_{m}$ твердых растворов на 145 градусов, кристаллографическая структура остается кубической без явных признаков ромбических искажений. 


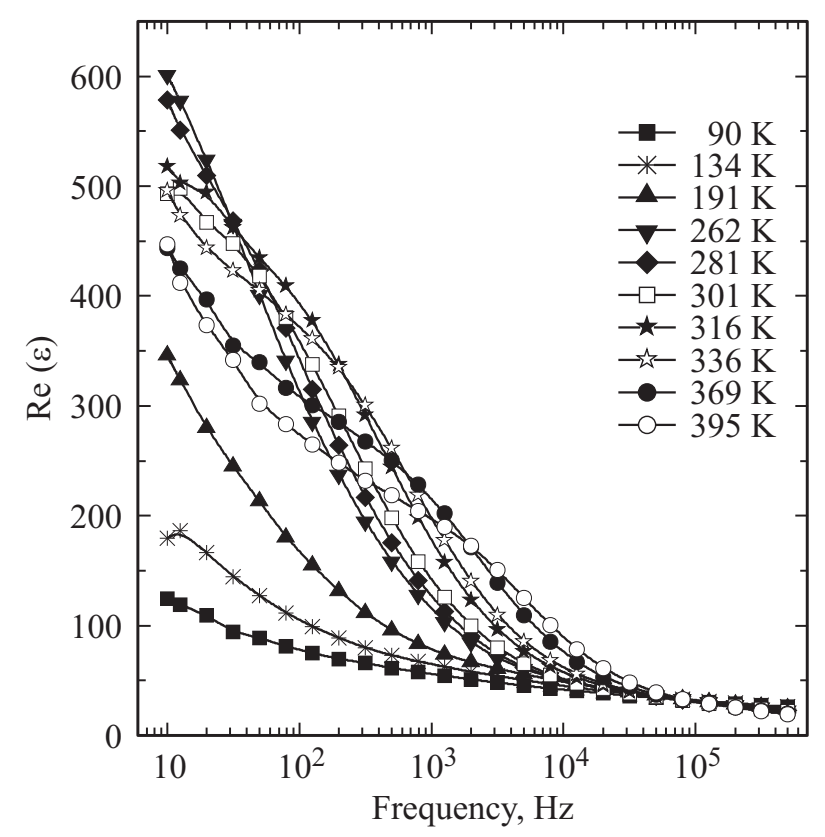

Pис. 3. Фрагмент спектров действительной части диэлектрической проницаемости $\varepsilon$ для состава с концентрацией $x=0.3$.

Измеренные температурные зависимости скорости звука $V_{L}$ составов с концентрацией $x=0.1,0.2$ и 0.3 представлены на рис. 4-6. Для состава с $x=0.1$ (рис. 4) наблюдается „скачок“ вниз скорости звука при температуре антиферродисторсионного (AFD)-перехода $T_{a}=255 \mathrm{~K}$, который существует у всех твердых растворов при $x \leq 0.1[34,35]$. Температура этого перехода увеличивается с ростом концентрации $x$ и существенно превышает температуру максимума диэлектрической

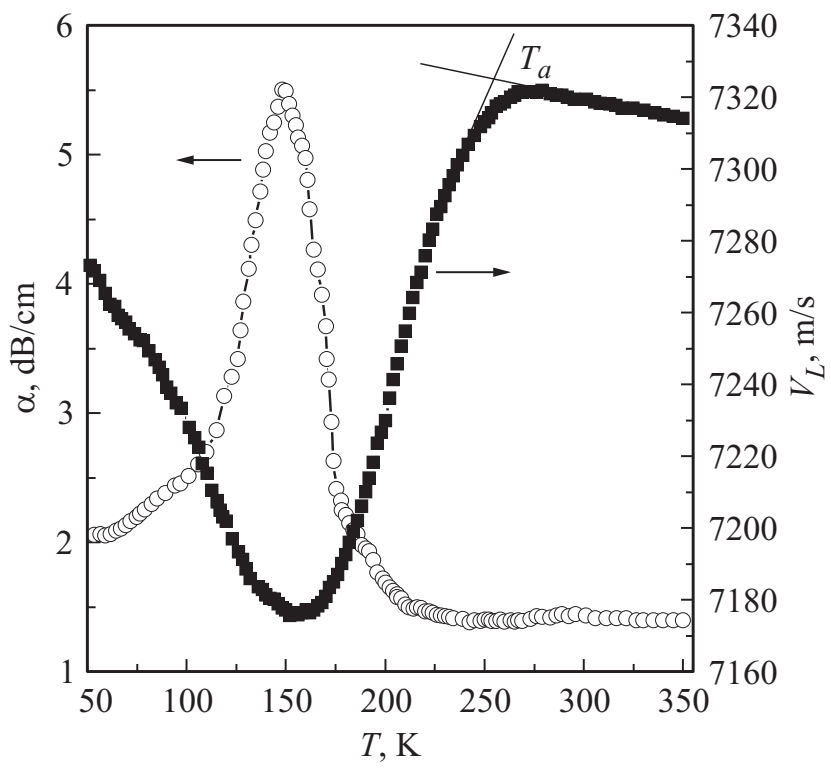

Рис. 4. Температурные зависимости скорости $V_{L}$ и затухания $\alpha$ продольной ультразвуковой волны для твердого раствора $0.9 \mathrm{SrTiO}_{3}-0.1 \mathrm{BiFeO}_{3}$. проницаемости $T_{m}$, определяющую в данном случае релаксорное состояние и равную $215 \mathrm{~K}(1 \mathrm{KHz})$ для $x=0.1$. Таким образом, у твердых растворов с концентраций феррита висмута $0<x \leq 0.1$ наблюдается сосуществование сегнетоэлектрической и антиферродисторсионных неустойчивостей при температурах ниже $T_{a}$. Температурная зависимость затухания ультразвуковой волны $\alpha(T)$ демонстрирует пик при „скачке“ скорости звука при AFD-переходе (рис. 4), что является характерной чертой фазовых переходов.

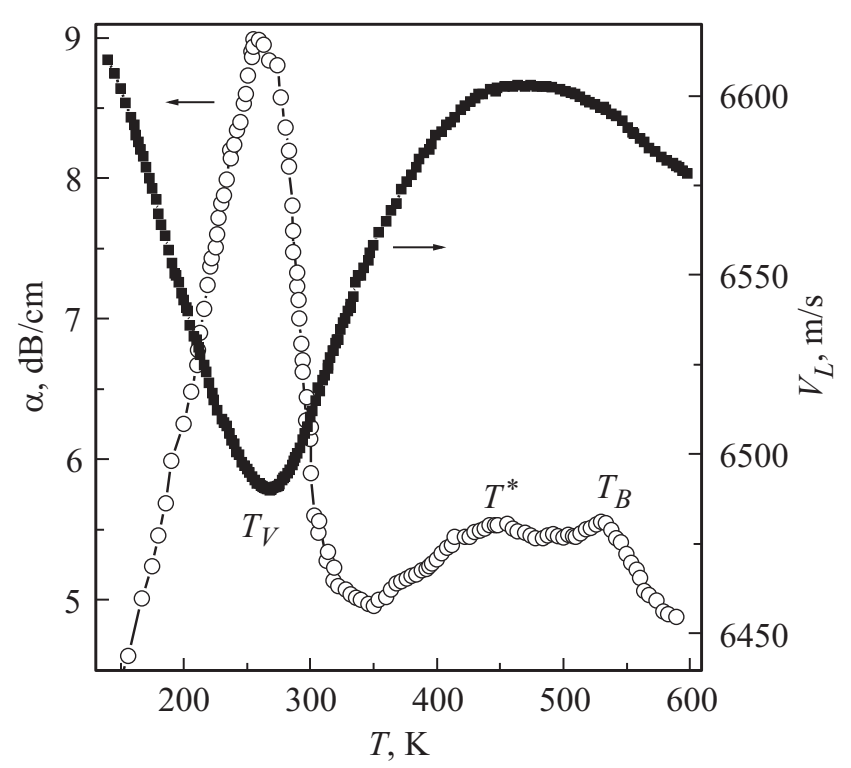

Рис. 5. Температурные зависимости скорости $V_{L}$ и затухания $\alpha$ продольной ультразвуковой волны для твердого раствора $0.8 \mathrm{SrTiO}_{3}-0.2 \mathrm{BiFeO}_{3}$.

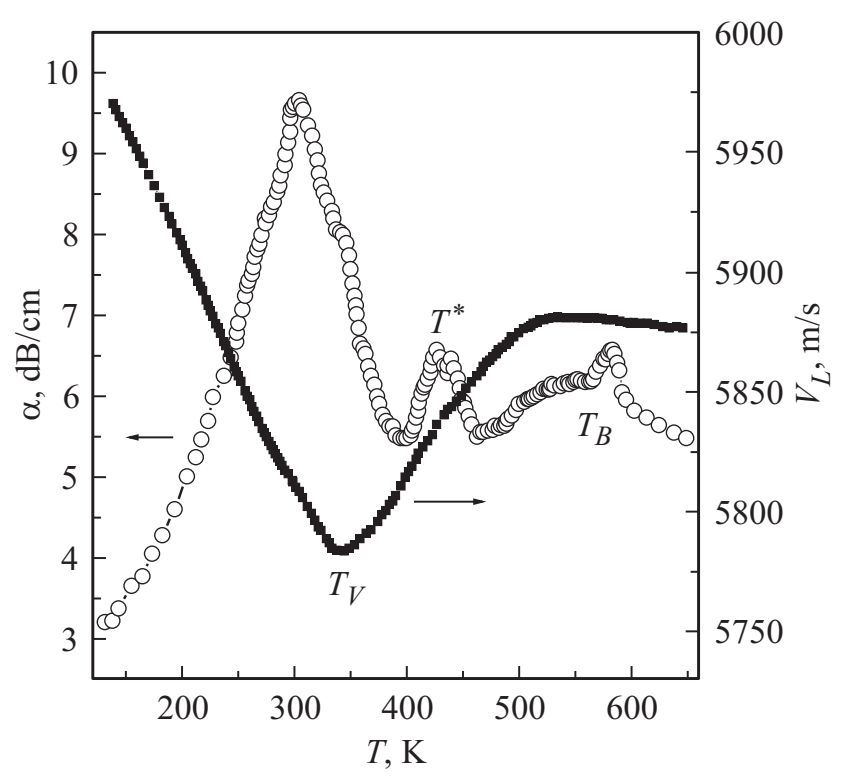

Рис. 6. Температурные зависимости скорости $V_{L}$ и затухания $\alpha$ продольной ультразвуковой волны для твердого раствора $0.7 \mathrm{SrTiO}_{3}-0.3 \mathrm{BiFeO}_{3}$. 
Следует заметить, что в классических релаксорах (например, в магнониобате свинца и твердых растворах на его основе) температура минимума зависимости скорости звука $T_{V}$, практически, совпадает с $T_{m}$ [43 и ссылки там же].

Именно такое поведение демонстрируют температурные зависимости скорости звука $V_{L}(T)$ для твердых растворов 0.8 STO-0.2 BFO и 0.7 STO-0.3 BFO (рис. 5,6). Кроме минимумов скорости, соответствующих релаксорному состоянию, у этих составов обнаружены пики затухания при температурах $T_{V}$, а также размытые максимумы затухания $\alpha(T)$ в высокотемпературном диапазоне 400-600 К. Как уже указывалось, пики затухания ультразвуковой волны наблюдались при температуре Бернса $T_{\mathrm{B}}$ и температуре $T^{*}$ в наиболее изученном релаксоре PMN (рис. 7). Выявленные высокотемпературные аномалии затухания звука подтверждают релаксорное состояние твердых растворов с $x=0.2$ и 0.3 , а также позволяют определить температуры Бернса $\left(T_{\mathrm{B}}=532\right.$ и $584 \mathrm{~K}$ соответственно для $x=0.2$ и 0.3$)$ и $T^{*}\left(T^{*}=450\right.$ и $430 \mathrm{~K}$ соответственно). Интересен тот факт, что при наличии диэлектрической релаксации, характерной для релаксоров, высокотемпературных пиков затухания у составов с $x \leq 0.1$ нами не обнаружено (рис. 4), что стимулирует дальнейшее исследование.

Известно, что соотношение Кюри-Вейсса выполняется у релаксоров для зависимостей $\varepsilon^{\prime}(T)$ при температуpax примерно на $150 \mathrm{~K}$ выше $T_{m}$ [39]. По температуре, при которой происходит отклонение кривой $1 / \varepsilon^{\prime}(T)$ от линейной зависимости, оценивалась температура $T_{d}$, которая рассматривалась в качестве температуры Бернса для измеренных составов [35]. Следует заметить, что

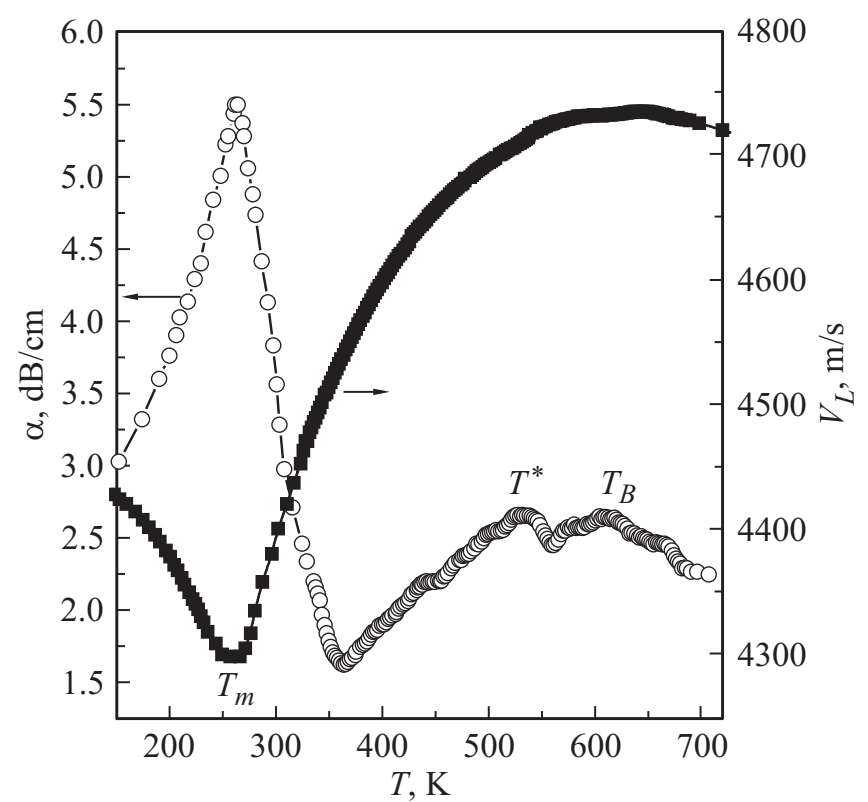

Pис. 7. Температурные зависимости скорости $V_{L}$ и затухания $\alpha$ продольной ультразвуковой волны для PMN при характерных температурах $T_{m}, T^{*}$ и $T_{\mathrm{B}}$.

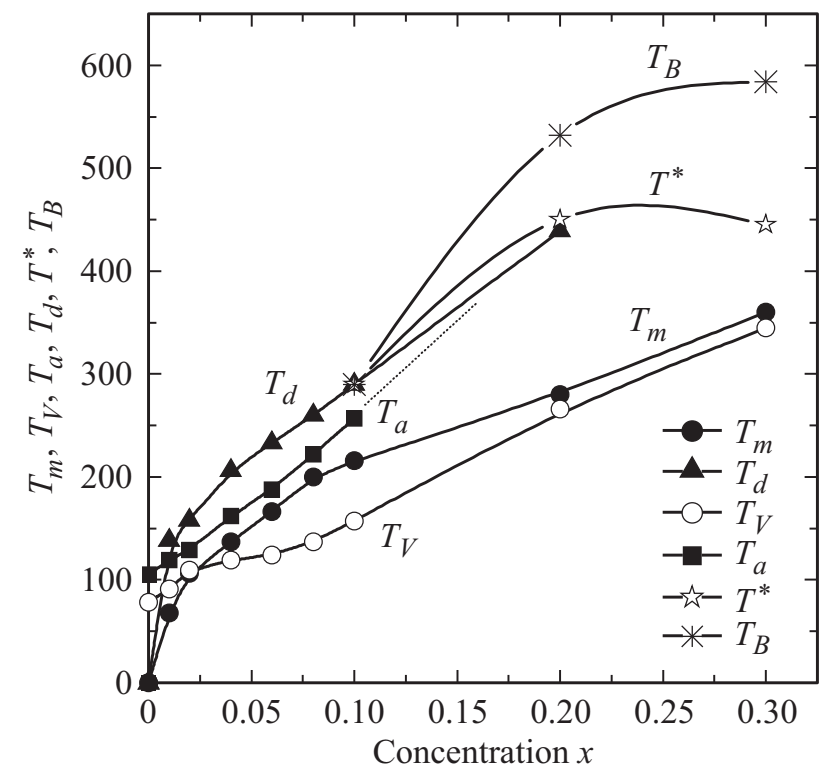

Рис. 8. Фрагмент фазовой диаграммы системы твердых растворов $(1-x) \mathrm{SrTiO}_{3}-x \mathrm{BiFeO}_{3}$. Приведены концентрационные зависимости $T_{V}(x), T_{m}(x), T_{d}(x)$, температуры антиферродисторсионного перехода $T_{a}$, температуры Бернса $T_{\mathrm{B}}$, а также $T^{*}$.

в силу нелинейности $1 / \mathcal{E}^{\prime}(T)$ величина $T_{d}$ зависит от диапазона температур измерения, превышающего $T_{m}$. Так, сравнение величины $T_{d}$, полученной для составов с $x=0.2$ и 0.3 , с величиной $T_{\mathrm{B}}$, определенной по аномалии затухания звука, указывает на приблизительность оценки температуры Бернса по результатам диэлектрических измерений.

Полученные акустические данные позволили уточнить фазовую диаграмму твердых растворов системы $(1-x) \mathrm{STO}-x$ BFO в той области концентраций $x$ и температур, при которых происходит подавление антиферродисторсионной неустойчивости, а релаксорное состояние характеризуется температурами Бернса и $T^{*}$ (рис. 8). Особенностью данной системы является тот факт, что, в диапазоне концентраций $0<x \leq 0.1$ при сосуществовании антиферродисторсинной и сегнетоэлектрической неустойчивостей, наблюдается только диэлектрическая релаксация, которая приписывается релаксорному состоянию. Явные признаки релаксорного состояния, включающие в себя, помимо диэлектрической релаксации, акустические аномалии при температурах Бернса и $T^{*}$ выявлены начиная с концентрации феррита висмута $x=0.2$.

\section{4. Заключение}

Таким образом, проведены систематические исследования системы твердых растворов $(1-x) \mathrm{SrTiO}_{3}-x \mathrm{BiFeO}_{3}$ при $0.1 \leq x \leq 0.3$ в широком температурном диапазоне. Обнаружены аномалии скорости и затухания звука при характерных для релаксорного 
состояния температурах $\left(T_{m}, T_{\mathrm{B}}\right.$ и $\left.T^{*}\right)$ для составов с $x>0.1$. На основании полученных экспериментальных данных уточнена фазовая диаграмма системы.

\section{Список литературы}

[1] J. Kuwata, K. Uchino, S. Nomura. Jpn. J. Appl. Phys. 21, 1298 (1982).

[2] S.W. Choi, T.R. Shrout, S.J. Jang, A.S. Bhalla. Ferroelectrics 100, 29 (1989).

[3] S.-E. Park, T.R. Shrout. J. Appl. Phys. 82, 1804 (1997).

[4] G. Xu. J. Phys. Soc. Jpn. 79, 011011 (2010).

[5] S. Zhang, F. Li. J. Appl. Phys. 111, 031301 (2012).

[6] Y. Liu, J.F. Scott, B. Dkhil1. APL Mater. 4, 064109 (2016).

[7] K.A. Müller, H. Burkard. Phys. Rev. B 19, 3593 (1979).

[8] W. Zhong, D. Vanderbilt. Phys. Rev. B 53, 5047 (1996).

[9] M.A. Saifi, L.E. Cross. Phys. Rev B 2, 677 (1970).

[10] H. Hemberger, M. Nicklas, R. Viana, R. Lunkenheimer, A. Loidl, R. Bohmer. J. Phys. Condens. Matter 8, 4673 (1996).

[11] A. Yamanaka, M. Kataoka, Y. Inaba, K. Inoue, B. Henlen, E. Courtens. Europhys. Lett. 50, 688 (2000).

[12] V.V. Lemanov, E.P. Smirnova, P.P. Sirnikov , E.A. Tarakanov. Phys. Rev. B 52, 315 (1996).

[13] В.В. Леманов, Е.П. Смирнова, Е.А. Тараканов. ФТТ 37, 2476 (1995).

[14] R. Wang, Y. Inaguma, M. Itoh. Mater. Res. Bull. 36, 1693 (2001).

[15] В.В. Леманов, Е.П. Смирнова, Е.А. Тараканов. ФТТ 39, 714 (1997).

[16] J.G. Bednorz, K.A. Müller. Phys. Rev. Lett. 52, 2289 (1984).

[17] U. Bianchi, J. Dec, W. Kleemann, J.G. Bednorz. Phys. Rev B 51, 8737 (1995).

[18] U. Bianchi, W. Kleemann, J.G. Bednotz. J. Phys. Condens. Matter 6, 1229 (1994).

[19] W. Kleemann, H. Schremmer. Phys. Rev. B 40, 7428 (1989).

[20] M.E. Guzva, V.V. Lemanov, P.A. Markovin, T.A. Shapligina. Ferroelectrics 218, 93 (1998).

[21] C. Ang, Z. Yu, P.M. Vilarinho, J.L. Baptista. Phys. Rev. B 57, 7403 (1998).

[22] Ю.Н. Веневцев, Г.С. Жданов, С.П. Соловьев. Кристаллография 5, (1960).

[23] С.В. Киселев, Р.П. Озеров, Г.С. Жданов. Докл. АН СССР 145, 1255 (1962).

[24] Yu.F. Popov, A.M. Kadomtsev, G.P. Vorobiev, A.K. Zvezdin. Ferroelectrics 162, 135 (1994).

[25] D. Lebeugle, D. Colson, A. Forget, M. Viret. Appl. Phys. Let. 91, 022907 (2007).

[26] D. Lebeugle, D. Colson, A. Forget, M. Viret, P. Bonville1, J.F. Marucco, S. Fusil. Phys. Rev. B 76, 024116 (2007).

[27] V.V. Shvartsman, W. Kleeman, R. Hanmount, J. Krisel. Appl. Phys. Lett. 90, 2115 (2007)

[28] J. Wang, J.B. Neaton, H. Zheng, V. Nagarajan, S.B. Ogale, B. Liu, D. Viehland, V. Vaithyanathan, D.G. Schlom, U.V. Waghmare, N.A. Spaldin, K.M. Rabe, M. Wuttig, R. Ramesh. Science 299, 1719 (2003).

[29] K.Y. Yun, D. Ricinschi, T. Kanashima, M. Okuyama. Jpn. J. Appl. Phys. 43, L647 (2004).

[30] V.R. Palkar, C. Kundaliya Darshan, S.K. Malik, S. Bhattacharya. Phys. Rev. B 69, 212102(3) (2004).

[31] V.A. Khomchenko, D.A. Kiselev, J.M. Viera, J. Li, A.L. Kholkin, A.M.L. Lopes, Y.G. Pogorelov, J.P. Araujo, M. Maglione. J. Appl. Phys. 103, 024105(6) (2008).
[32] B. Kundys, A. Maignan, C. Martin. Appl. Phys. Lett. 92, 3, 112905 (2008).

[33] H. Naganuma, N. Shimura, J. Miura, H. Shima, S. Yasui, K. Nishida, T. Katoda, T. Iijima, H. Funakubo, S. Okamura. J. Appl. Phys. 103, 314 (2008).

[34] Е.П. Смирнова, А.В. Сотников, Н. Schmidt, Н.В. Зайцева, M. Weihnacht. ФTT 51, 2348 (2009).

[35] Е.П. Смирнова, А.В. Сотников, Н.В. Зайцева, Н. Schmidt, M. Weihnacht. ФTT 56, 960 (2014).

[36] G. Burns, B.A. Scott. Solid State Commun. 13, 423 (1973).

[37] G. Burns, F.H. Dacol. Solid State Commun. 48, 853 (1983).

[38] G. Burns, F.H. Dacol. Phys. Rev. B 28, 2527 (1983).

[39] D. Vieland, J.F. Li, S.J. Jang, L.E. Cross, M. Wuttig. Phys. Rev. B 46, 8013 (1992).

[40] E.P. Smirnova, A.V. Sotnikov, O.E. Kvyatkovskii, M. Weihnacht, V.V. Lemanov. J. Appl. Phys. 101, 84117 (2007).

[41] B. Dkhil, P. Gemeiner, A. Al-Barakaty, L. Bellaiche, E. Dul'kin, E. Mojaev, M. Roth. Phys. Rev. B 80, 064103 (2009).

[42] E. Dul'kin, M. Roth, P.-E. Janolin, B. Dkhil. Phys. Rev. B 73, 012102 (2006).

[43] E. Smirnova, A. Sotnikov, S. Ktitorov, N. Zaitseva, H. Schmidt, M. Weihnacht. J. Appl. Phys. 115, 054101 (2014).

[44] E. Smirnova, S. Smirnov, A. Sotnikov, H. Schmidt, M. Weihnacht. Ferroelectrics 469, 67 (2014).

[45] A. Nabereznov, S. Vakhrushev, B. Donner, D. Strauch, H. Modden. Eur. Phys. J. B 11, 13 (1999).

[46] K. Hirota, Z.-G. Ye, S. Wakimoto, P.M. Gehring, G. Shirane. Phys. Rev. B 65, 104105 (2002).

[47] G. Xu, G. Shirane, J.R.D. Copley, P.M. Gehring. Phys. Rev. B 69, 064112 (2004).

[48] P.M. Gehring, S.-E. Park, G. Shirane. Phys. Rev. Lett. 84, 5216 (2000).

[49] P.M. Gehring, S.-E. Park, G. Shirane. Phys. Rev. B 63, 224109 (2001).

[50] G. Shirane, P.M. Gehring. J. Phys. Soc. Jpn. 70, Suppl. A, 227 (2001).

[51] T.-Y. Koo, P.M. Gehring, G. Shirane, V. Kiryukhin, G. Lee, S.-W. Cheong. Phys. Rev. B 65, 144113 (2002).

[52] Е.Г. Фесенко. Семейство перовскитов и сегнетоэлектричество. Атомиздат, М. (1972). 248 с. 